\title{
Review Paper: Aspects related to Resilience in People with Spinal Cord Injury
}

\author{
Amir Rahmani Rasa ${ }^{1}$, *Seyyed Ali Hosseini ${ }^{1}$, Hojjatollah Haghgoo ${ }^{1}$, Hamid Reza Khankeh ${ }^{2}$, G.G. Ray ${ }^{3}$
}

1. Department of Occupational Therapy, University of Social Welfare and Rehabilitation Sciences, Tehran, Iran.

2. Department of Nursing, University of Social Welfare and Rehabilitation Sciences, Tehran, Iran.

3. Indian Institiute of Tecnology Bombay, Mumbai, India.

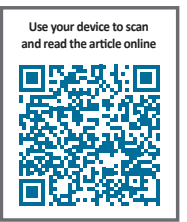

citation: Rahmani Rasa A, Hosseini SA, Haghgoo H, Khankeh HR, Ray GG. [Aspects Related to the Resilience in People With Spinal Cord Injury (Persian)]. Archives of Rehabilitation Journal. 2017; 17(4):350-359. http://dx.doi.org/ $10.21859 /$ jrehab-1704350

http://dx.doi.org/ 10.21859/jrehab-1704350

Received: 1 Jun. 2016 Accepted: 7 Oct. 2016

Keywords: Spinal cord injury, Resilience, Adaptation

\section{ABSTRACT}

Objective Rehabilitation of the spinal cord injury is a continuous process needing adaptation in all aspects of life. Successful adaptation to challenging situations is called "resilience". Occupational therapists can help the person reconstruct his/ her identity and adapt by improving their resilience. It is also extensive and diverse in concept and definition. It is extremely influenced by the culture and environment. This study aims to narratively review the evidences published on the aspects related to the resilience of people with spinal cord injury.

Materials \& Methods Comprehensive electronic search of keywords including "spinal cord injury", "resilience" and "adaptation" was done via Google Scholar, PubMed, Scopus, ScienceDirect and Pro Quest and IranMedex to find related articles published within 2000-2015 according to inclusion criteria. From the initially obtained 1823 articles, 1756 articles were omitted due to failure to satisfy the inclusion criteria or being repetitions. After actors underlying resilience and 4 and 5 articles focused on social participation and psychosocial adaptation after spinal cord injury in respect.

Results As seen from the included documents, there are various definitions of resilience. Resilience was defined as coping with the conditions after spinal cord injury such that the person takes in his/ her activities of daily living with improved mental health and appropriate community mobility. It is also defined as the process that involves a complex interaction of the person with his/ her environment. There are four trajectories in terms with resilience. Fifty percent of people involved are resilient, 25 percent of them showed recovery, 12.8 percent showed delayed distress, and 12.5 percent have chronic dysfunction. This adaptation is definitely influenced by the surrounding social context since any environmental complication can lead to a decline in resilience. The more the environmental relations are, the simpler adaptation occurs. So, it is necessary to notice the communicative and environmental simplifiers in the rehabilitation program. Family and economic status have a significant role in the resilience of people with spinal cord injury. Resilient people with spinal cord injury have better adaptation and lower levels of depression while entering rehabilitation as well as show more acceptance of the disability during discharge and more satisfaction of their lives. The resilience is extensively impacted by the individual characteristics and social circumstances. The resilience is a multidimensional concept that includes contextual factors, social participation, and mental adaptation. Being a male, having a mild-to-moderate physical job, having a high academic level before the injury, and obtaining appropriate education after injury can lead to resilience and paid employment later. In contrast, misunderstanding of physical complications, passive personality, pessimism, and lack of psychosocial support from the family are barriers for being employed after injury. The less the family and environmental barriers are, the better mental health and adaptation is anticipated. Factors such as job and suitable education before the injury as well as optimism and positive attitude provide effective resilience later. Spirituality and self-control have a good prognosis for resilience. The supportive role of the environment, particularly of that of family members, is also important. Conclusion Resilience in spinal cord injury needs psychologically more attempt than the physical aspects, because it needs more time to adapt after a sudden accident. Spirituality, stress management strategies, optimism, and social support from family members and friends facilitate resilience. As resilience is impacted by the culture and environment, more studies on the explanation of the aspects related to the resilience of Iranian population is recommended.

\section{* Corresponding Author: \\ Seyyed Ali Hosseini, PhD \\ Address: Department of Occupational Therapy, University of Social Welfare and Rehabilitation Sciences, Koodakyar Ave, Daneshjoo Blvd, Evin, Tehran, Iran. Tel: +98 (21) 22180037}

E-Mail: alihosse@gmail.com 


\title{
مطالعه مرورى: جنبههاى مرتبط با تابآورى در افراد با ضايعه نخاعى
}

\author{
امير رحمانىرسا'، "سيدعلى حسينى'، حجتالله حقكو'، حميدرضا خانكه'، جى جى رى'

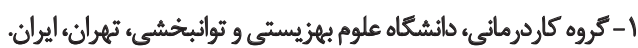

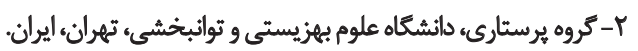

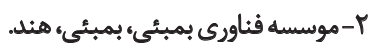

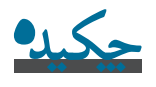

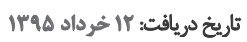

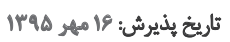

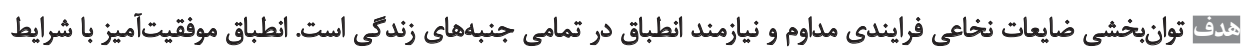

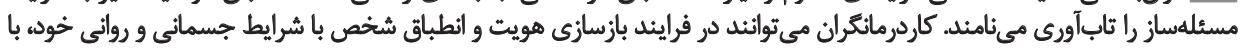

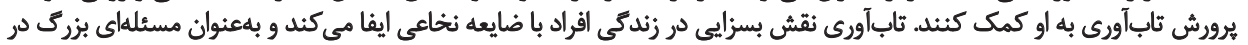

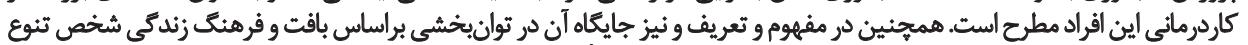

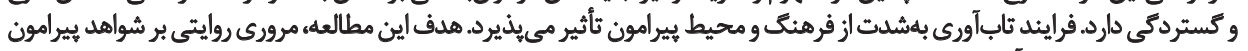

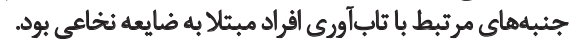

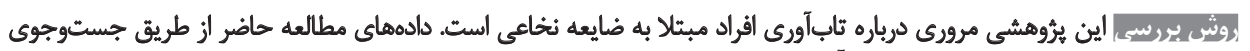

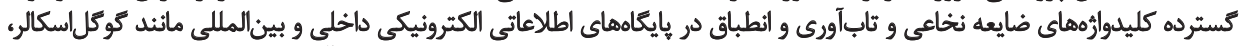

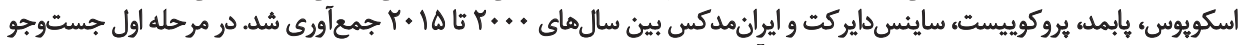

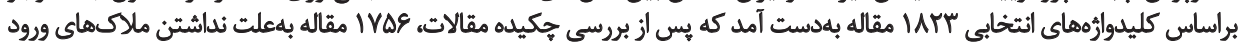

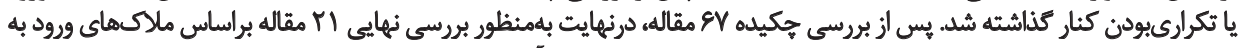

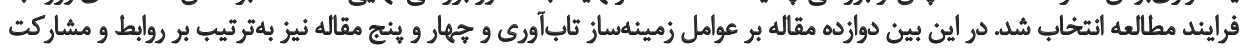

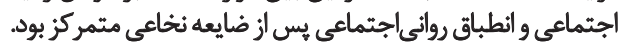

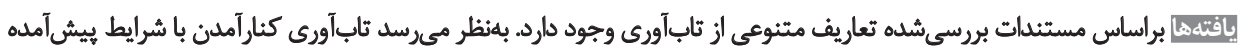

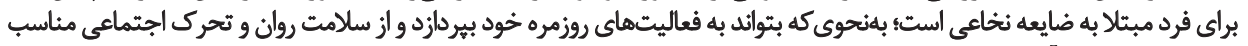

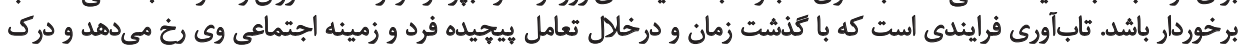

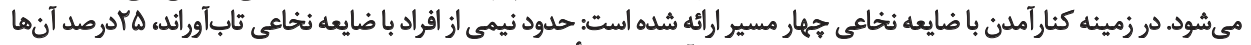

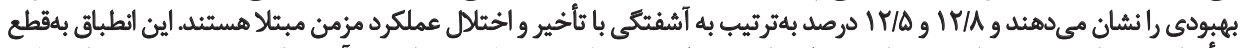

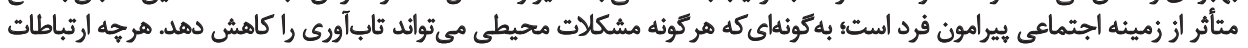

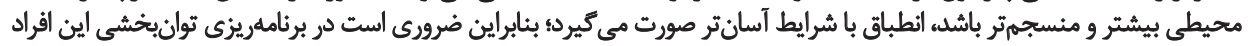

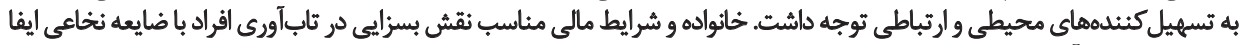

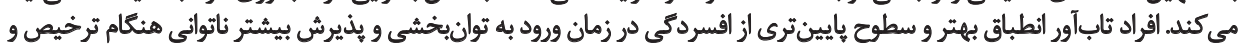

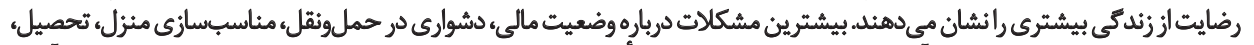

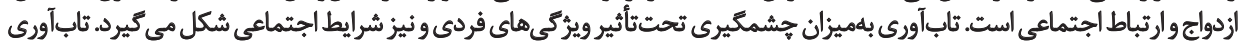

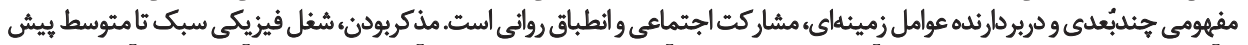

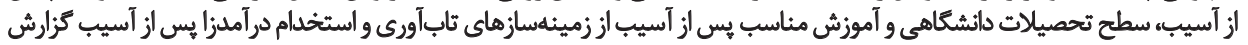

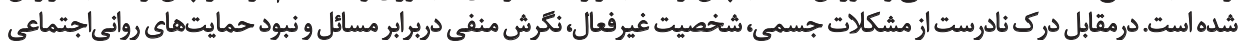

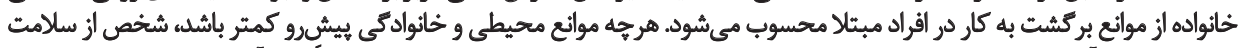

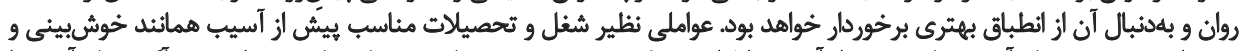

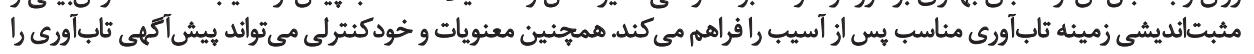

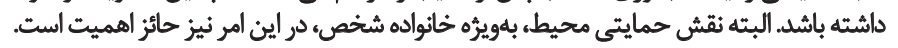

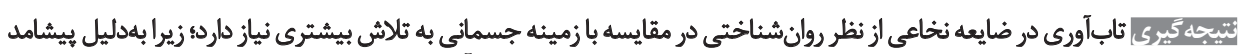

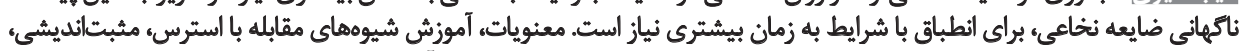

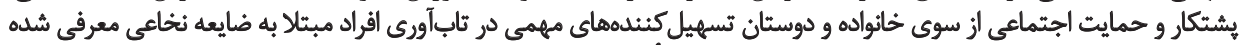

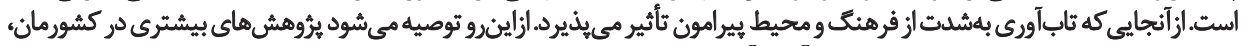

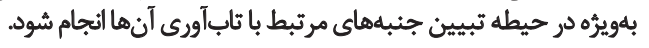

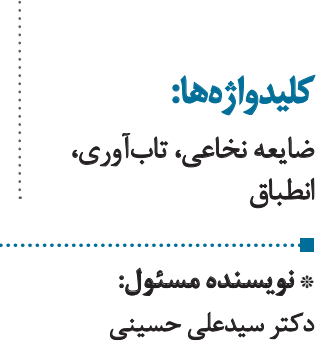

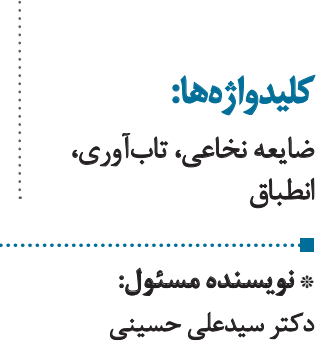

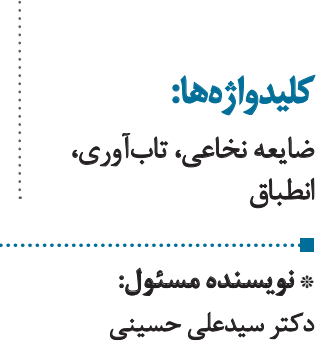
-

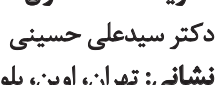

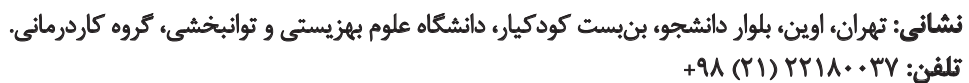
alihosse@gmail.com رايانامهن
\end{abstract}


كاردرمانكران هم توانيد در فرايند بازسازى هويت و انطباق بيمار

مقدمه

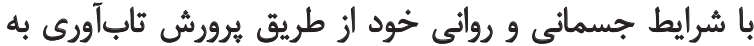

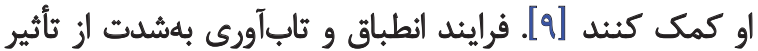

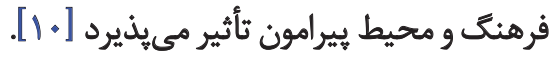

در زمينه تابآورى بيشتر مطالعات به ابعاد جسمانى تابآورى بآئا

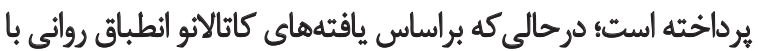

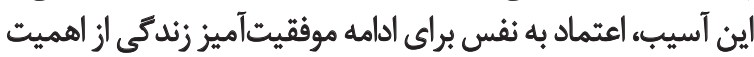

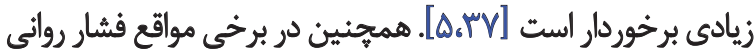

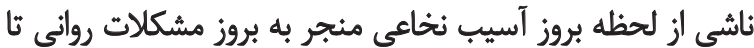

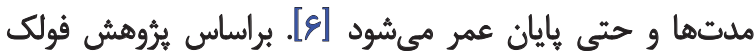

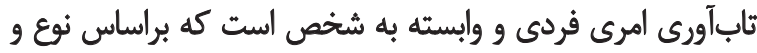

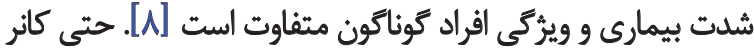

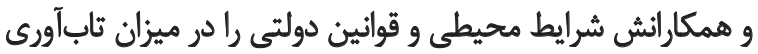

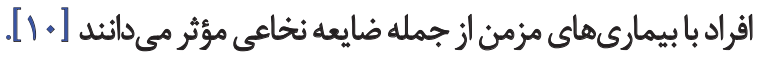

تابآورى نقش بسرايى در زندگى افراد با ضايعه نخاعى ايفا

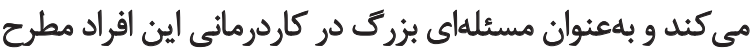

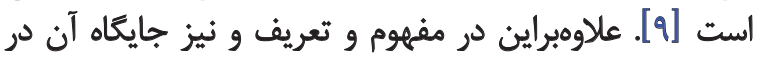

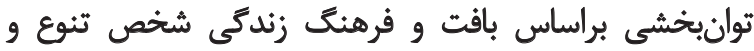

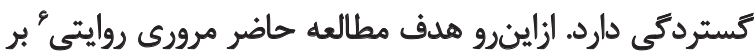

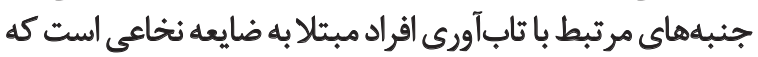

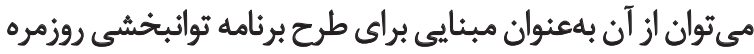

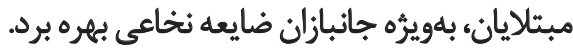

$$
\text { روشّ بروسى }
$$

Spi- با جستوجوى كسترده الكترونيكى كليدوارههاى دal Cord Injury (SCl), Resilience و Adaptation

6. Narrative review

2. Paraplegia

3. Tetraplegia

4. Resilience

5. Occupational therapists

جدول ا. كليدوازهمايى درباره تابآورى افراد با ضايعه نخاعى برمبناي مِ

Spinal Cord Injury (SCI), Resilience and occupational therapy (OT), Physical adaptation, Resilience at home, Adaptation methods, Occupational therapy and resilience, Resilience program, Resilience and contexts, Social resilience, Personal factor, Environmental factor, Family adaptation, $\mathrm{SCl}$ and family, OT and resilience, Activities of Daily Living (ADL), ADL at home, $A D L$ and resilience, $A D L$ at home.

توانبخننى

جدول r. ملاكهاي ورود وخروج مطالعه.

مراكنهاى خروج

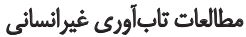

مطالعات بهله زبان غير ازئ فارسيى و انغليسيى
ملاكيهاى ورود

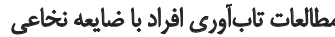

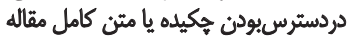

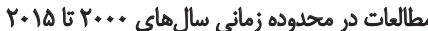




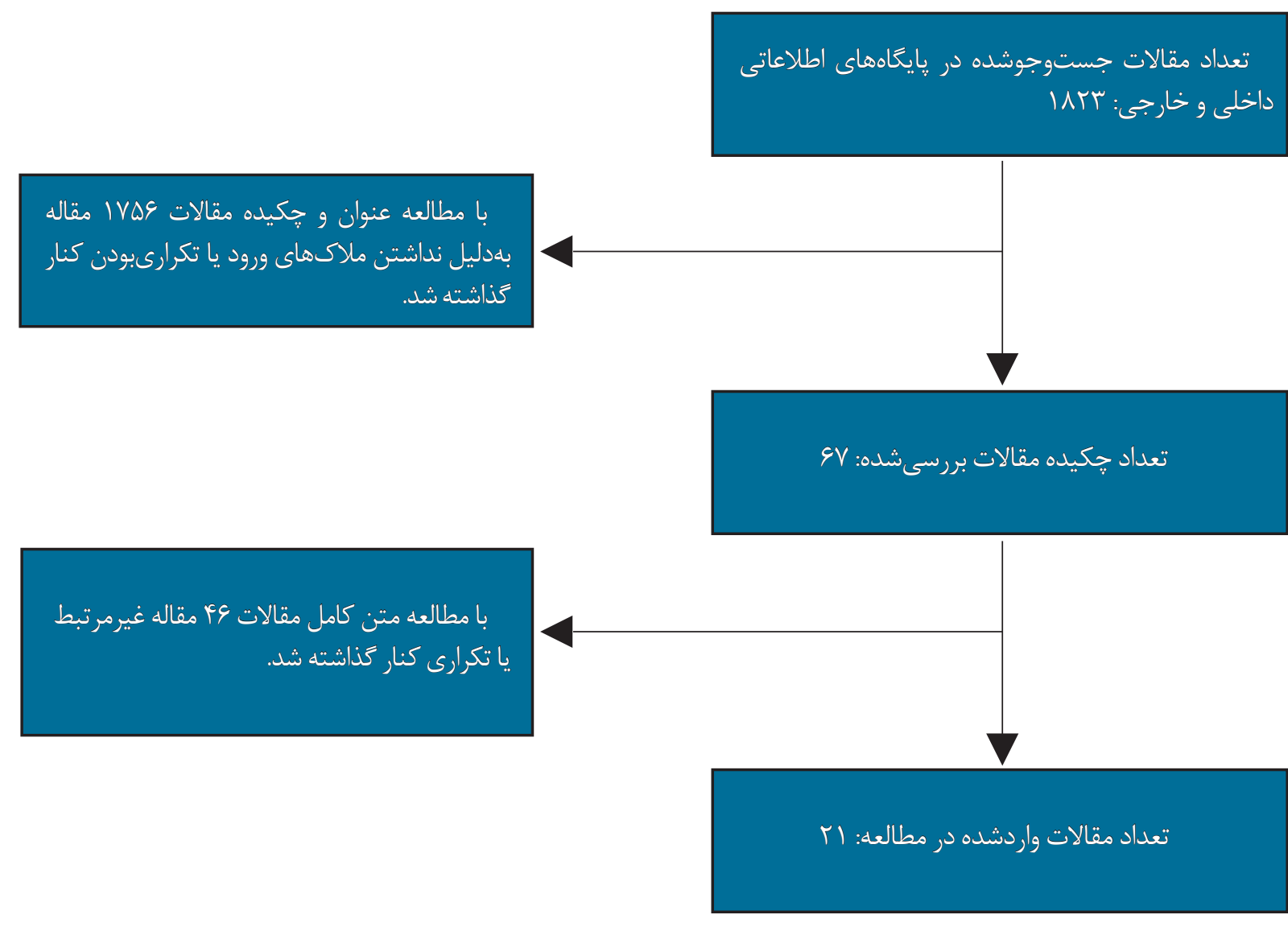

توانبخننى

تصوير ا.فلوجارت مطالعه.

دوازده مقاله بر عوامل زميئهساز تابآورى و جهار و وينج مقاله نيز

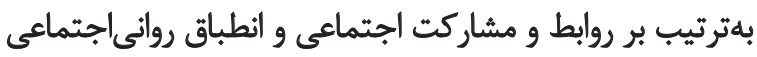

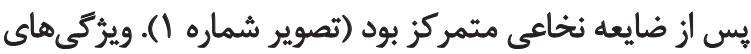
مقالات بررسى شده در جدول شماره بـ آمده است.

$$
\text { بحث مفوم ثابآورى }
$$

در متون مختلف تعاريف متعدد و متنوعى از تابآورى ارائه

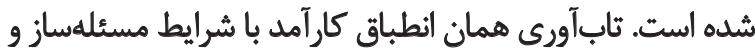

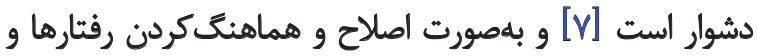

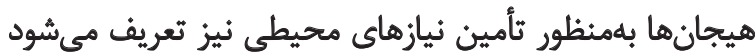

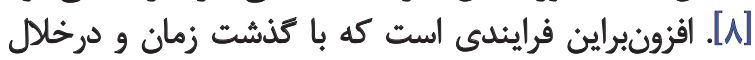

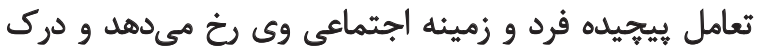

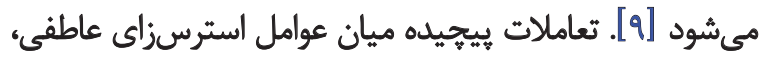

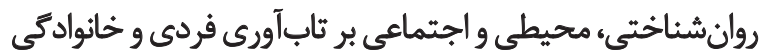

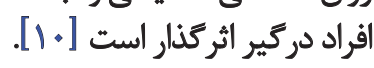

يايكاههاى اطلاعاتى داخلى و بينالمللى نظير اسكويوس"، يابمد،؛

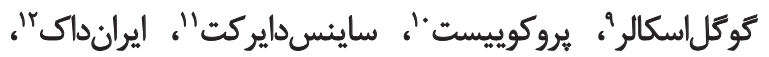

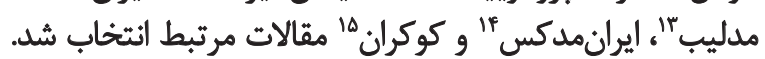

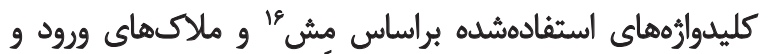

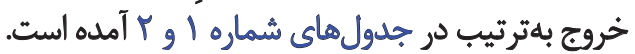

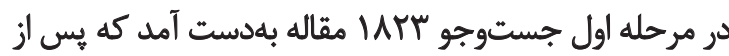

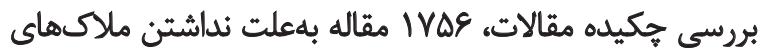

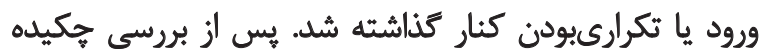

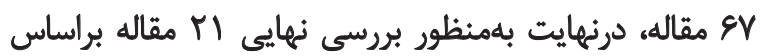

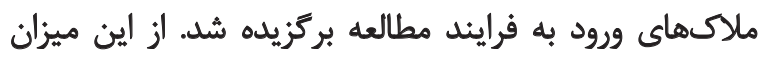

\section{Scopus}

8. Pubmed

9. Google Scholar

10. Pro Quest

11. Science Direct

12. Iran DOC

13. Medlib

14. Iran Medex

15. Cochrane Data Base of Systematic Reviews 16. MESH 
جدول با. مشخصات مطالعات بروسي شده داراى ملاك ورود.

\begin{tabular}{|c|}
\hline \\
\hline 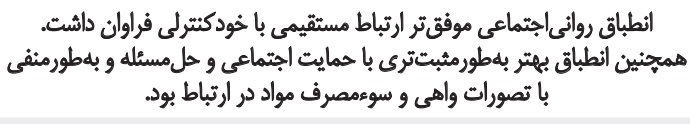 \\
\hline 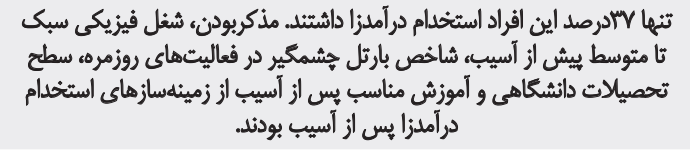 \\
\hline 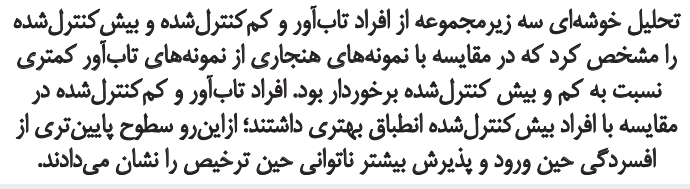 \\
\hline 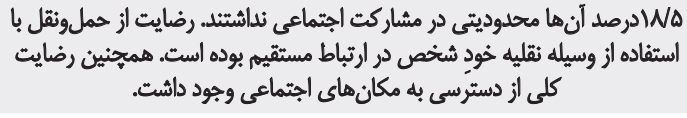 \\
\hline دوابط با ديكران در ايجاد \\
\hline 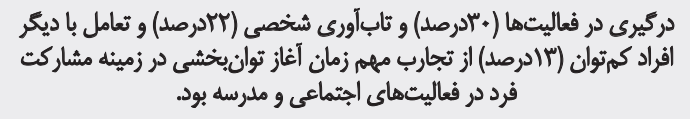 \\
\hline
\end{tabular}

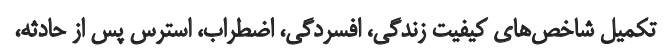

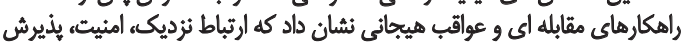

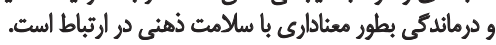

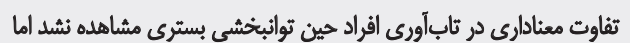

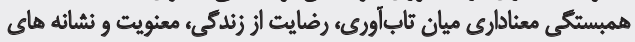

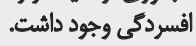

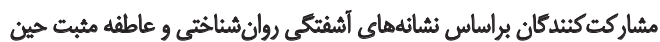

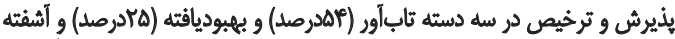

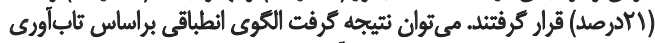

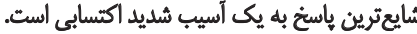

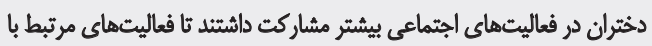

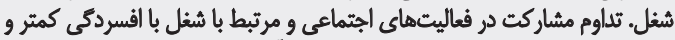

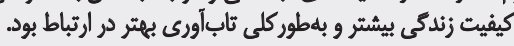

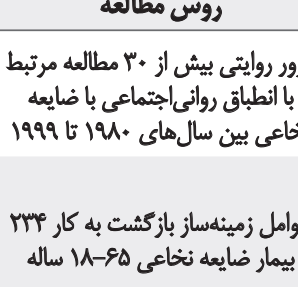

نوع مطالعه

نويسنده

سال

براسي نمونهاي شخصيتي 199 فرد

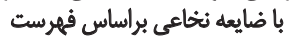
إبعاد شخصيثى ينيجعاملى فئي

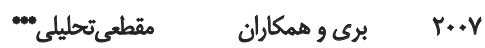

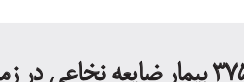

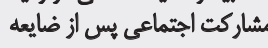

F...

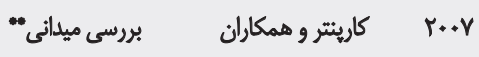

روابط فردى يس الز ضايعه نخاعى در أ

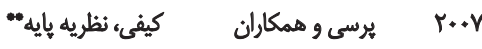

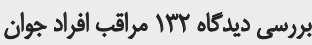

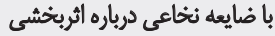

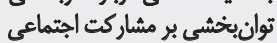
أنها

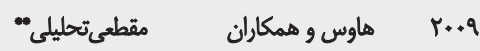

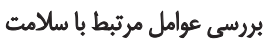

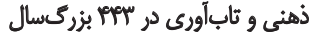

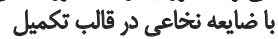
كزارش هائ خودسئجى دابح

تاب آورى و شاخصمهاى انطباق حين

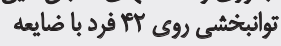

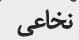

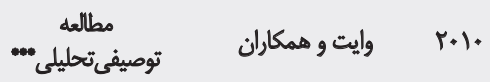

بروسى مقطعى *A فرد با ضايعه

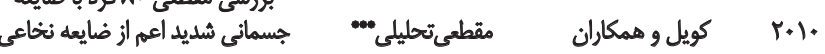
وضريه

بررسى مشاركت الجتماعى و بيامدهايى

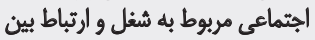

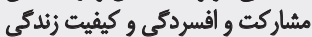

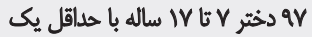

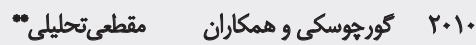

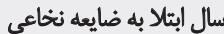

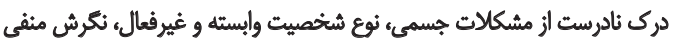

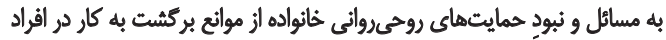

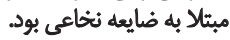

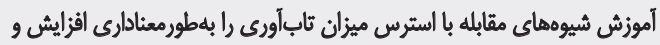

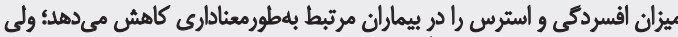

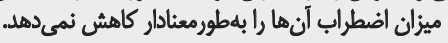

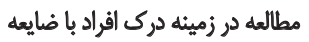

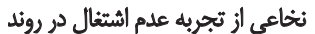
بركشت به كار

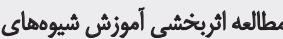

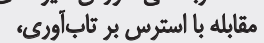

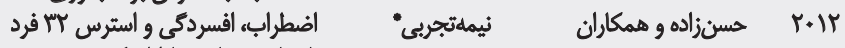

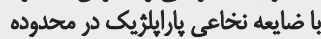
سنى بآ تا عات سال

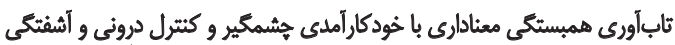

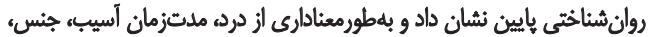

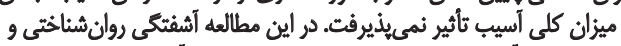

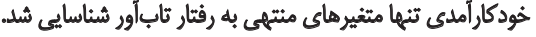

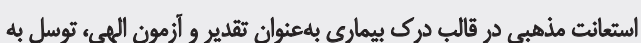

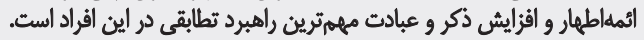

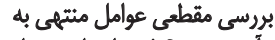

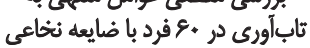

هطالعه در زمينه راهبرد تطابقى مهيم

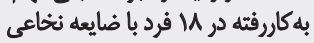

: T.11

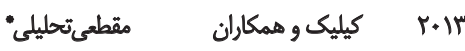

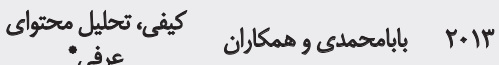




\begin{tabular}{|c|c|c|c|c|}
\hline ثتايج مطالعه & روش مطالعه & نوع مطالعه & نويسنده & سال \\
\hline 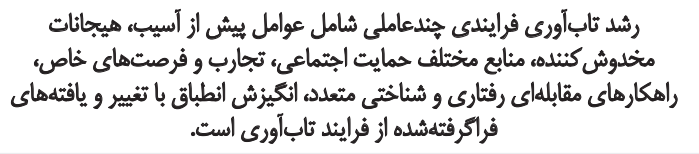 & 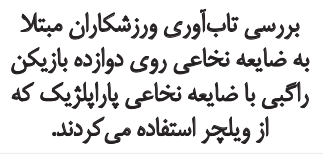 & كيفى، هديلدشثناسي" & ماجيدا و همكاران & $r \cdot 1 r$ \\
\hline 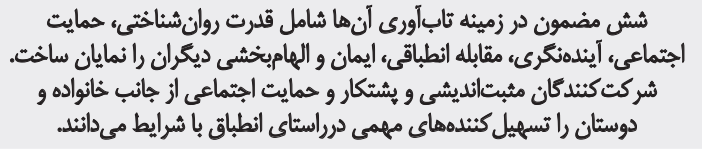 & 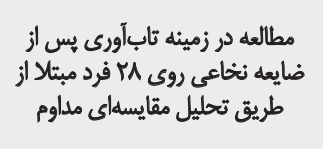 & كيفى، هيليدششناسي" & موندن و همكاران & $r+1 F$ \\
\hline 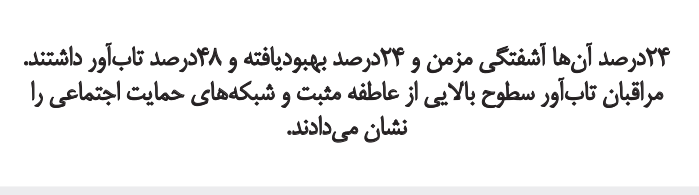 & 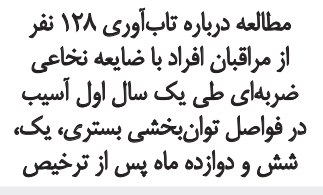 & "توصيفى تحليلي" & اليوت و همكاران & $r \cdot 1 f$ \\
\hline 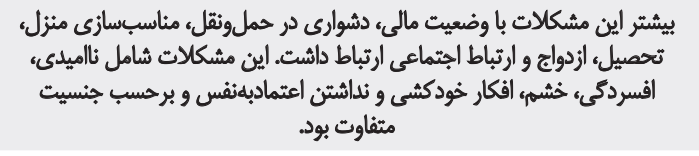 & 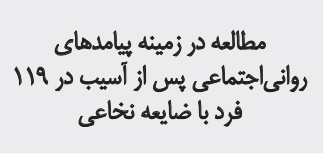 & "وصيفى مقطعى ״ت" & خزايعيورو و همكاران & $r=1 f$ \\
\hline 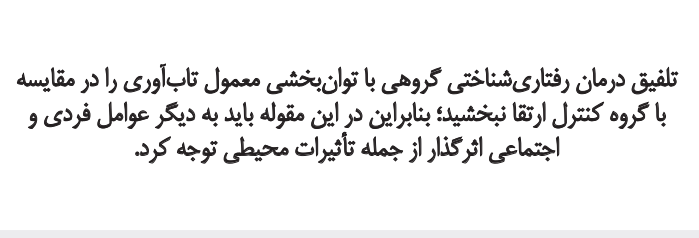 & 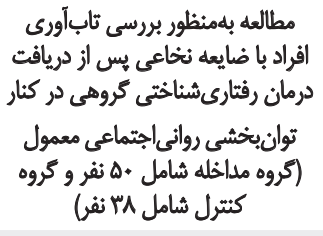 & كوهورت" & كست و همكاران & $r \cdot 10$ \\
\hline 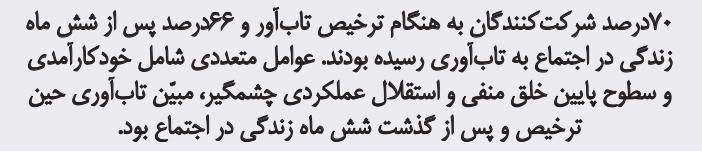 & 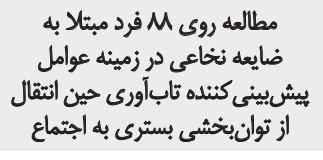 & مطالعه طولى" & كست و همكاران & $r \cdot 10$ \\
\hline 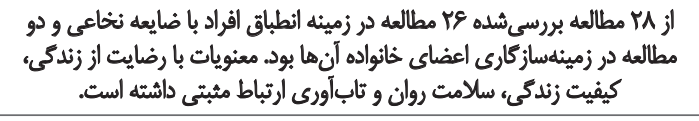 & 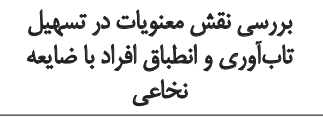 & هرودى" & جونز و همكاران & $r \cdot 1 \Delta$ \\
\hline توانبخنتنى & & \multicolumn{3}{|c|}{ 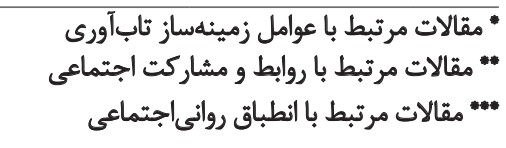 } \\
\hline
\end{tabular}

فردى و نيز شرايط اجتماعي شكل مي كيرد. ثاب آورى هفهومي

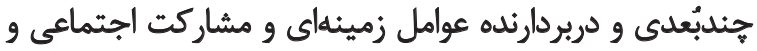
انطباق روانى أست. عوامل زمينهساز تابآورى مذكربودنء شُغل فيزيكي سيك نا متوسط بيش ازئ آسيب،

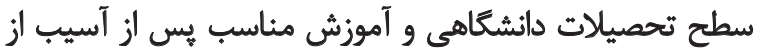

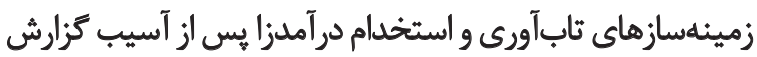

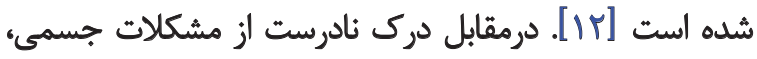

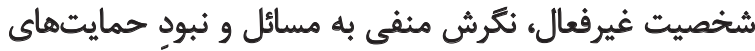

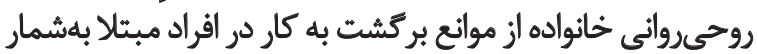

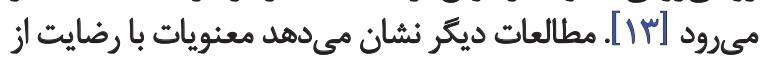

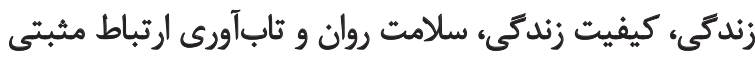

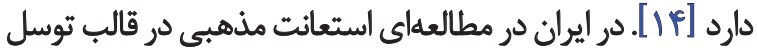

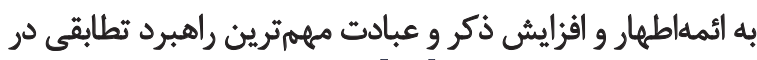

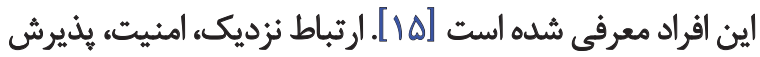

بونائو و كويل جههار مسير " را در زميئه كنارآمدن با ضايعه

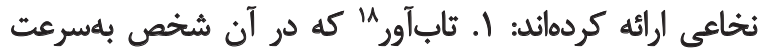

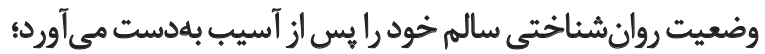

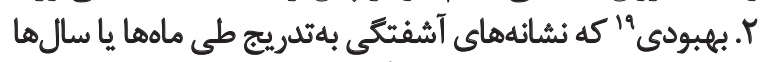

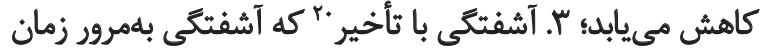

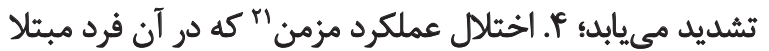

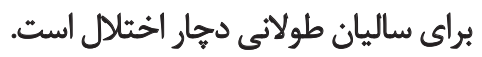
درحدود نيمى از افراد با ضايعه نخاعى ثاب آوراند، هابرصد

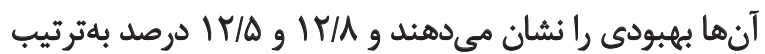

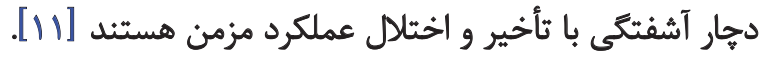

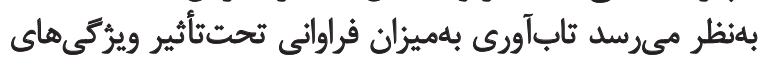

17. Trajectory

18. Resilient

19. Recovery

20. Delayed distress

21. Chronic dysfunction 


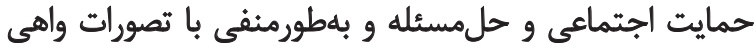

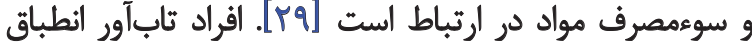

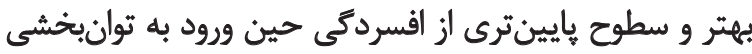

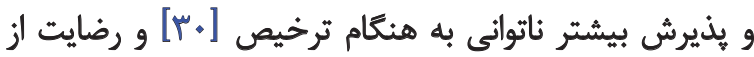

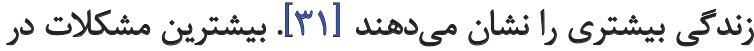

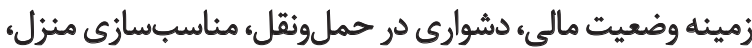

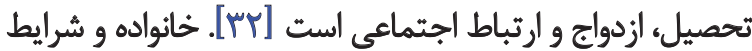

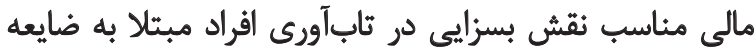

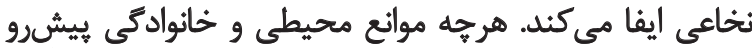

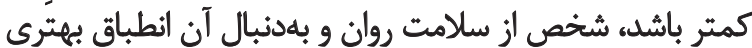

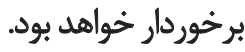

نتيجلمَيرى

همان طور كه از مطالب ذكرشده نمايان است، تعاريف متنوعى از

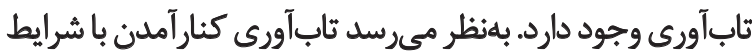

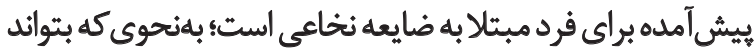

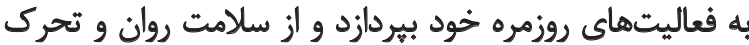

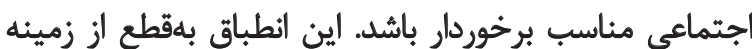

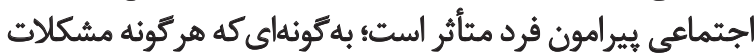

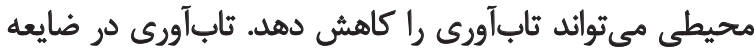

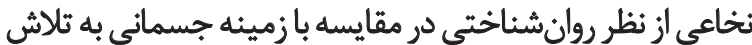

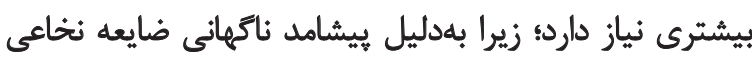

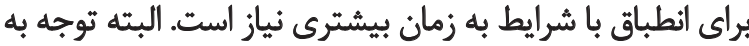

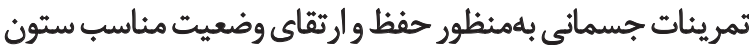

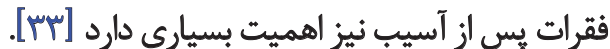
معنويات، آموزش شيوههاى مقابله با استرس، مثبتانديشى،

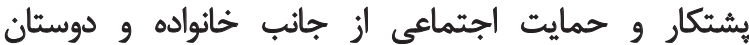

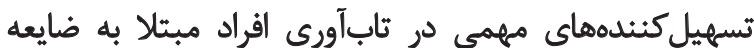

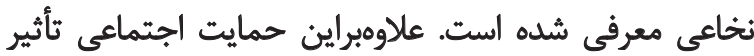

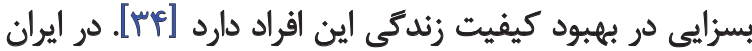

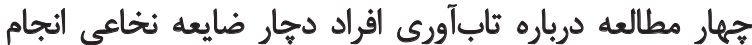

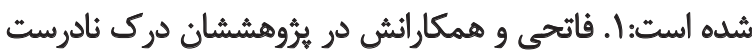

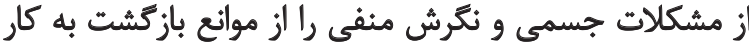

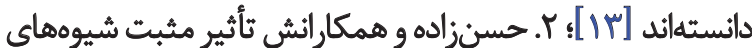

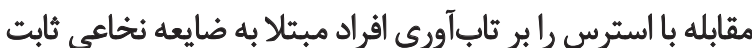

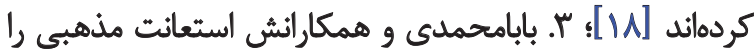

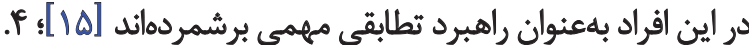

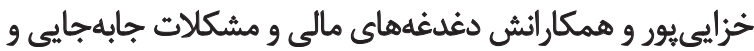

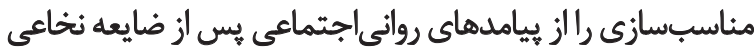

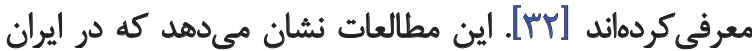

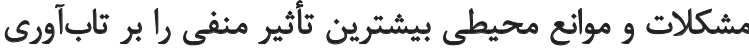

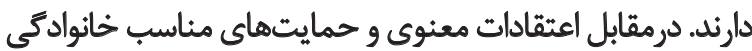
نقش بسزايى در ارتقاى انطباق شخص با شرايط ايفا مى كند. در

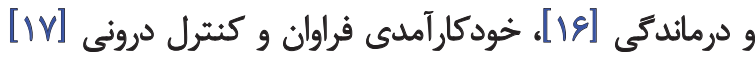

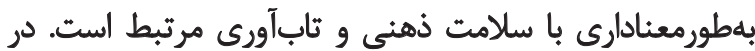

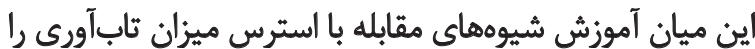

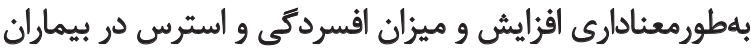

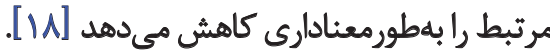

مثبتانديشى و يشتكار و حمايت اجتماعى از جانب خانواده

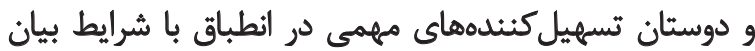

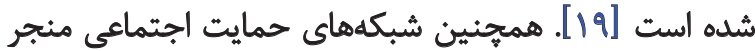

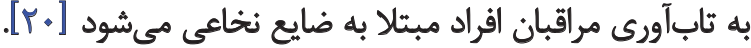

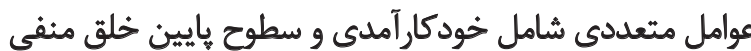

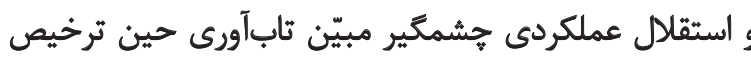

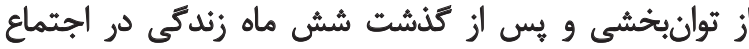

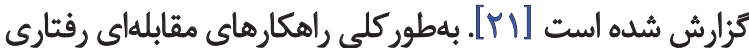

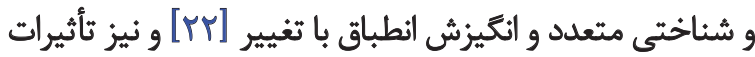

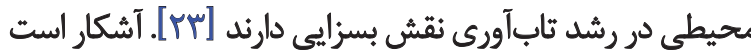

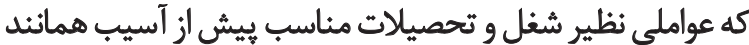

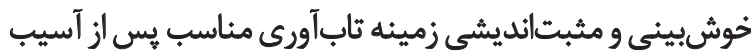

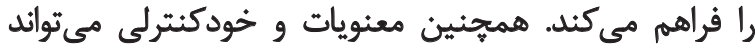

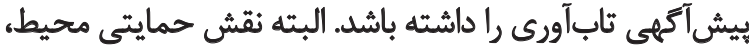

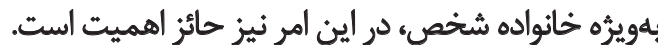

روابط و مشاركت الجتماعى يس از ضايعه افراد جوان باضايعه نخاعى دركيرشدن "تر فر فعاليتها از ابتداي

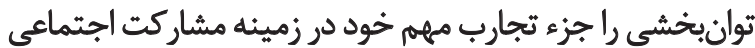

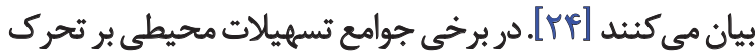

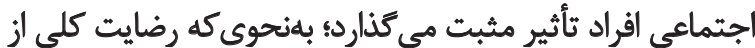
دسترسى به مكانهاى اجتماعى وجود دارد [بهائ.

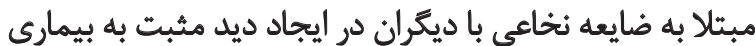

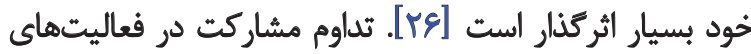

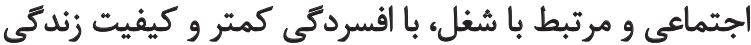

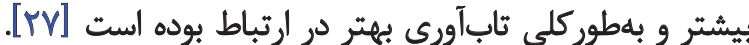

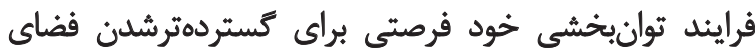

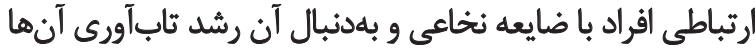

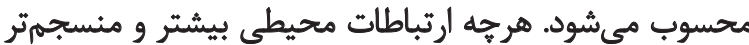

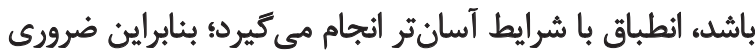

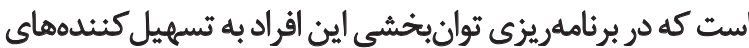
محيطى وارتباطى توجه شود. انطباق روانى اجتماعى يس از ضايعه مطالعات حاكى از آن است كه يك الكوى انطباقي براساس

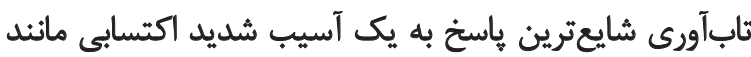

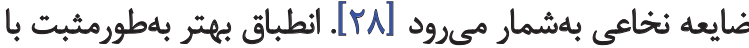




\section{References}

[1] Umphred DA, Lazaro RT, Roller M, Burton G. Neurological rehabilitation. Philadelphia: Elsevier Health Sciences; 2013.

[2] Somers MF. Spinal cord injury: functional rehabilitation. New Jersey: Prentice Hall; 2001.

[3] Mingaila S, Krisciūnas A. Occupational therapy in spinal cord injury. Medicina. 2003; 40(8):816-19. PMID: 15300005

[4] Van den Berg M, Castellote J, Mahillo-Fernandez I, de PedroCuesta J. Incidence of spinal cord injury worldwide: a systematic review. Neuroepidemiology. 2010; 34(3):184-92. doi: $10.1159 / 000279335$

[5] Catalano D, Chan F, Wilson L, Chiu CY, Muller VR. The buffering effect of resilience on depression among individuals with spinal cord injury: a structural equation model. Rehabilitation Psychology. 2011; 56(3):200-11. doi: 10.1037/a0024571

[6] Krause JS, Newman S. Posttraumatic stress disorder and spinal cord injury. Archives of Physical Medicine and Rehabilitation. 2010; 91(8):1182-87. doi: 10.1016/j.apmr.2010.05.012

[7] Windle G. What is resilience? A review and concept analysis. Reviews in Clinical Gerontology. 2011; 21(2):62-159. doi: 10.1017/ s0959259810000420

[8] Folke C, Carpenter SR, Walker B, Scheffer M, Chapin T, Rockström J. Resilience thinking: integrating resilience, adaptability and transformability. Ecology and Society. 2010; 15(4):20.

[9] Pendleton HM, Schultz-Krohn W. Pedretti's occupational therapy: practice skills for physical dysfunction. Philadelphia: Elsevier Health Sciences; 2013.

[10] Connor KM, Davidson JR. Development of a new resilience scale: The Connor-Davidson resilience scale (CD-RISC). Depression \& Anxiety. 2003; 18(2):76-82. doi: 10.1002/da.10113

[11] Bonanno GA, Kennedy P, Galatzer-Levy IR, Lude P, Elfström ML. Trajectories of resilience, depression, and anxiety following spinal cord injury. Rehabilitation Psychology. 2012; 57(3):236-42. doi: $10.1037 /$ a 0029256

[12] Tomassen P, Post M, Van Asbeck F. Return to work after spinal cord injury. Spinal Cord. 2000; 38(1):6-24. doi 10.1038/ sj.sc.3100948

[13] Fatehi F, Kamali M. [Perceived experiences of unemployed people with spinal cord injury in the process of returning to work (Persian)]. Research in Rehabilitation Sciences. 2011; 8(2):254-62.

[14] Jones K, Simpson GK, Briggs L, Dorsett P. Does spirituality facilitate adjustment and resilience among individuals and families after SCI? Disability and Rehabilitation. 2015; 15(1):830-8.

[15] Babamohamadi H, Negarandeh R, Dehghan Nayeri N. [Important Coping Strategies Used by Individuals with Spinal Cord Injury: A Qualitative Study (Persian)]. Journal of Qualitative Research in Health Sciences. 2013; 2(1):90-100.

[16] Migliorini C, Tonge B. Reflecting on subjective well-being and spinal cord injury. Journal of Rehabilitation Medicine. 2009; 41(6):445-50.

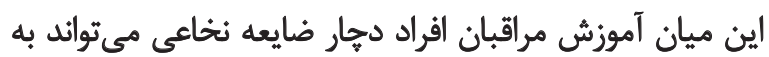

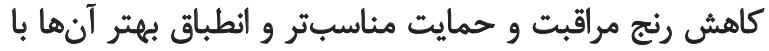

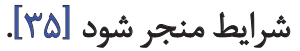

Leosgeres

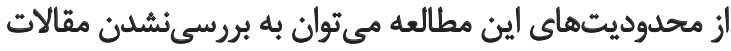

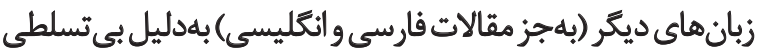

$$
\begin{aligned}
& \text { به آن زبان ها اشاره كرد. } \\
& \text { Wolsing }
\end{aligned}
$$

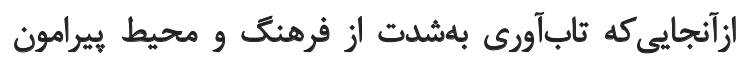

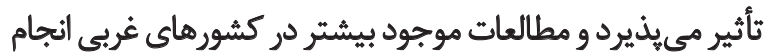

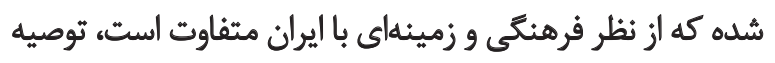

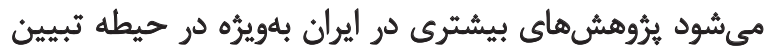

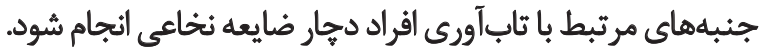

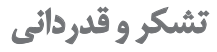

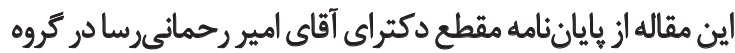

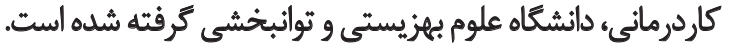


[17] Kilic S, Dorstyn D, Guiver N. Examining factors that contribute to the process of resilience following spinal cord injury. Spinal Cord. 2013; 51(7):553-57. doi: 10.1038/sc.2013.25

[18] Hasanzadeh PS, Zare H, Alipor A. [The efficacy of stress inculation training (SIT) on resilience, anxiety, depression and stress among spinal cord injury patients (Persian)]. Journal of Jahrome University of Medical Sciences. 2012; 10(3):590-98.

[19] Monden K, Trost Z, Catalano D, Garner A, Symcox J, Driver S, et al. Resilience following spinal cord injury: A phenomenological view. Spinal Cord. 2014; 52(3):197-201. doi: 10.1038/sc.2013.159

[20] Elliott TR, Berry JW, Richards JS, Shewchuk RM. Resilience in the initial year of caregiving for a family member with a traumatic spinal cord injury. Journal of Consulting and Clinical Psychology. 2014; 82(6):1072-078. doi: 10.1037/a0037593

[21] Guest R, Craig A, Tran Y, Middleton J. Factors predicting resilience in people with spinal cord injury during transition from inpatient rehabilitation to the community. Spinal Cord. 2015; 53(9):682-86. doi: 10.1038/sc.2015.32

[22] Machida M, Irwin B, Feltz D. Resilience in competitive athletes with spinal cord injury the role of sport participation. Qualitative Health Research. 2013; 23(8):1054-065. doi: $10.1177 / 1049732313493673$

[23] Guest R, Craig A, Nicholson Perry K, Tran Y, Ephraums C, Hales A, et al. Resilience following spinal cord injury: A prospective controlled study investigating the influence of the provision of group cognitive behavior therapy during inpatient rehabilitation. Rehabilitation Psychology. 2015; 60(4):311-21. doi: $10.1037 /$ rep0000052

[24] House L, Russell H, Kelly E, Gerson A, Vogel L. Rehabilitation and future participation of youth following spinal cord injury: caregiver perspectives. Spinal Cord. 2009; 47(12):882-86. doi: $10.1038 / \mathrm{sc} .2009 .64$

[25] Carpenter C, Forwell SJ, Jongbloed LE, Backman CL. Community participation after spinal cord injury. Archives of Physical Medicine \& Rehabilitation. 2007; 88(4):427-33. doi: 10.1016/j. apmr.2006.12.043

[26] Pearcey TE, Yoshida KK, Renwick RM. Personal relationships after a spinal cord injury. International Journal of Rehabilitation Research. 2007; 30(3):209-19. doi: 10.1097/ mrr.0b013e32829fa3c1

[27] Gorzkowski JA, Kelly EH, Klaas SJ, Vogel LC. Girls with spinal cord injury: Social and job-related participation and psychosocial outcomes. Rehabilitation Psychology. 2010; 55(1):58-65. doi: $10.1037 / \mathrm{a} 0018275$

[28] Quale AJ, Schanke AK. Resilience in the face of coping with a severe physical injury: a study of trajectories of adjustment in a rehabilitation setting. Rehabilitation Psychology. 2010; 55(1):1200-208. doi: 10.1037/a0018415

[29] Livneh H. Psychosocial adaptation to spinal cord injury: The role of coping strategies. Journal of Applied Rehabilitation Counseling. 2000; 31(2):3-10.

[30] Berry JW, Elliott TR, Rivera P. Resilient, undercontrolled, and overcontrolled personality prototypes among persons with spinal cord injury. Journal of Personality Assessment. 2007; 89(3):292302. doi: $10.1080 / 00223890701629813$

[31] White B, Driver S, Warren AM. Resilience and indicators of adjustment during rehabilitation from a spinal cord injury. Rehabilitation Psychology. 2010; 55(1):230-38. doi: 10.1037/a0018451

[32] Khazaeipour Z, Norouzi-Javidan A, Kaveh M, Khanzadeh Mehrabani F, Kazazi E, Emami-Razavi SH. Psychosocial outcomes following spinal cord injury in Iran. Journal of Spinal Cord Medicine. 2014; 37(3):338-45. doi: 10.1179/2045772313y.0000000174

[33] Raeisi-dehkordi M, Sadeghi H, Bani-Talebi E, Ali-Akbarian A. [The comparison of traditional exercises and body weight supported training exercise on sensory-motor function, quality and quantity of walking in paraplegic spinal cord injured persons (Persian)]. Journal of Rehabilitation. 2015; 15(4):22-31.

[34] Khosravi-Tapeh N, Raheb G, Arshi M, Eglima M. [The relationship evaluation between physical diabled`s social support and life quality in Raad`s education centers (Persian)]. Journal of Rehabilitation. 2015; 16(2):176-185.

[35] Mohammadi S, Roshanzadeh M. [The effect of educational interventions based on Orem self-care model on family caregivers 'burden of patients with spinal cord injuries (Persian)]. Journal of Rehabilitation. 2016; 17(1):52-61.

[36] Lashani L, Farhoudian A, Azkhosh M, Dolatshahee B, Saadati H. Acceptance and Commitment Therapy in the Satisfaction of Veterans with Spinal Cord Injuries Spouses. Iranian Rehabilitation Journal. 2013; 11(2):56-60.

[37] Sadrossadat S J, Sadrossadat L. The Role of Self-Esteem on Vocational Rehabilitation of People with Spinal Cord Injury. Iranian Rehabilitation Journal. 2007; 5(1):56-59 
